\section{Cureus}

\title{
Laparoscopic Allograft Spacer Placement to Minimize Bowel Dose During Re-irradiation with Interstitial Brachytherapy
}

\author{
Shraddha Dalwadi ${ }^{1}$, Anuj Suri ${ }^{2}$, Aparna Kamat ${ }^{2}$, E. Brian Butler ${ }^{3}$, Andrew M. Farach ${ }^{3}$ \\ 1. Radiation Oncology, Baylor College of Medicine, Houston, USA 2. Obstetrics and Gynecology, Houston \\ Methodist Hospital, Houston, USA 3. Radiation Oncology, Houston Methodist Hospital, Houston, USA
}

$\square$ Corresponding author: Andrew M. Farach, amfarach@houstonmethodist.org

\section{Abstract}

In primary or re-irradiation of gynecologic malignancies, achieving optimal dosimetry with adjacent normal tissue becomes challenging. Surgical spacers are tissue-equivalent materials placed within the patient to protect organs at risk from long-term radiation effects and are commonly used in prostate cancer. We report the use of an allograft mesh to protect adhesed bowel from high-dose radiation for definitive treatment of recurrent endometrial cancer.

An 88-year-old female was diagnosed with International Federation of Gynecology and Obstetrics (FIGO) stage II endometrial cancer after she developed urinary frequency, hesitancy, and hematuria. She underwent neoadjuvant chemoradiation, followed by laparoscopic hysterectomy with bilateral salpingo-oophorectomy and adjuvant vaginal cuff brachytherapy. She developed $1.8 \mathrm{~cm}$ bilateral vaginal cuff recurrence and was dispositioned for interstitial brachytherapy. An allograft mesh spacer was placed laparoscopically before repeat, high dose rate brachytherapy to protect nearby structures. Dose-escalation was achieved without compromising normal tissue constraints. The patient tolerated the procedure without evidence of long-term toxicity at one year.

Multidisciplinary discussion may help identify patients who would benefit from spacer placement before select dose-escalated radiation therapy. Laparoscopic allograft mesh is one of many types of surgical spacers available for such patients.

Categories: Radiation Oncology, Oncology, Obstetrics/Gynecology

Keywords: brachytherapy

Received 09/24/2019

Review began 10/15/2019

Review ended 10/17/2019

Published 10/22/2019

(C) Copyright 2019

Dalwadi et al. This is an open access article distributed under the terms of the Creative Commons Attribution License CC-BY 3.0., which permits unrestricted use, distribution, and reproduction in any medium, provided the original author and source are credited.

\section{Introduction}

With high dose per fraction brachytherapy or stereotactic body radiation therapy, protecting adjacent normal tissue from high dose radiation is challenging and can be dose-limiting. Implanted spacing devices have been utilized to overcome normal tissue constraints and allow for dose-escalation. The first reported use of spacers in radiation therapy was in 1984, when a synthetic pelvic spacer was utilized to spare intestinal loops in the treatment of abdominal malignancies. In the same year, an oral spacer was used to minimize the risk of osteonecrosis $[1,2]$. Since then, many studies have reported the use of spacers in various anatomical sites. A variety of materials for surgical spacers exist, such as silicone or acrylic resin, blood patch, balloon, collagen, hydrogel, hyaluronic acid, saline, and acellular human dermis [3-5]. 
Currently, no guidelines exist for the use of spacers in radiotherapy. Reports in the literature describe the use of these techniques to minimize normal tissue dose in head and neck, cervical, and prostate cancer [6-8]. While short-term follow-up shows favorable safety profiles, longterm data is not presently available to evaluate the delayed complications of prophylactic spacer use in brachytherapy. However, in select cases where dose delivery to tumor remains suboptimal without compromising normal structure limits, surgical implants may decrease the risk of post-procedure morbidity without conceding oncologic outcome.

We present an elderly patient with a solitary vaginal cuff recurrence after previous chemoradiation. Acellular dermal matrix spacer was successfully placed laparoscopically at the time of interstitial high-dose rate brachytherapy to minimize bladder and small bowel dose.

\section{Case Presentation}

An 88-year-old female was diagnosed with International Federation of Gynecology and Obstetrics (FIGO) stage II endometrial cancer after she developed mixed urinary frequency and hesitancy with hematuria. Performance status at diagnosis was Eastern Cooperative Oncology Group (ECOG) 1. A cervical mass was identified on speculum exam. Biopsy confirmed poorlydifferentiated adenocarcinoma of cervical versus endometrial origin. Histopathologic analysis showed negative carcinoembryonic antigen, focally positive vimentin, patchy $\mathrm{p} 16$, and negative synaptophysin staining, favoring endometrial primary despite negative estrogen receptor staining. Staging computerized tomography (CT) was negative for metastatic disease. Given the bulk of disease in the cervix and lower uterine segment, neoadjuvant chemoradiation was favored for downstaging. Simulation was performed supine with full and empty bladder for adequate target coverage with motion. Subsequently, planning followed using threedimensional conformal technique. She began weekly cisplatin and pelvis radiation to $45 \mathrm{~Gy}$. However, treatment course was complicated by intractable diarrhea and subsequent dehydration requiring multiple hospitalizations. Treatment was ultimately suspended after four cycles and $30.6 \mathrm{~Gy}$.

Despite an interrupted course, magnetic resonance imaging (MRI) demonstrated a favorable treatment response and laparoscopic total hysterectomy with bilateral salphingooopherectomy was performed. Final pathology showed a 5-cm FIGO grade 2 endometrioid endometrial adenocarcinoma with $0.1 / 0.9 \mathrm{~cm}$ of myometrial invasion and no lymphovascular invasion. Margins were negative. Extensive tumor necrosis and treatment effect was present throughout, including in the cervical stroma, consistent with pre-treatment stage II disease.

Post-operatively, she was treated with high-dose rate vaginal cuff brachytherapy (24 Gy in four fractions prescribed to the surface at $0.5 \mathrm{~cm}$ depth of the upper $4 \mathrm{~cm}$ of the vagina), given her incomplete course of pelvic external beam radiation therapy. She tolerated treatment well without major complication and was followed closely for surveillance.

At six months, her speculum exam revealed 2-cm abnormality in the area of the vaginal cuff, within the high-dose brachytherapy field (Figures 1,2). Biopsy showed recurrent endometrial adenocarcinoma. Positron emission tomography with CT (PET-CT) and pelvic MRI showed no evidence of metastatic disease, only a PET-CT avid 1.8-cm nodular irregularity in the location of the biopsy-proven recurrence. Her case was discussed in a multi-disciplinary setting and the recommendation was made to proceed with local interstitial brachytherapy to allow for coverage of the recurrence and improved dose distribution in comparison to intracavitary techniques. 


\section{Cureus}

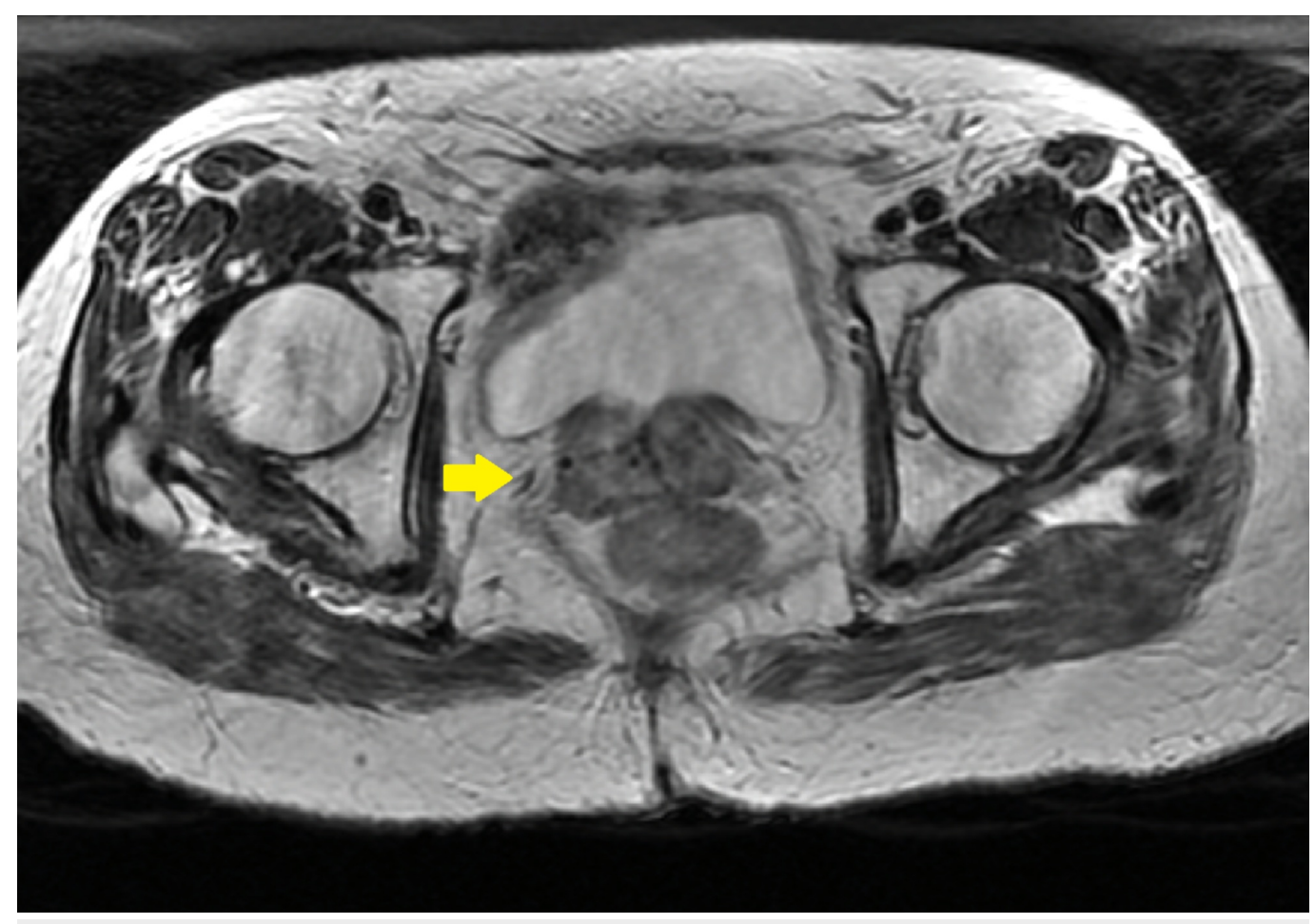

FIGURE 1: Pre-implant axial magnetic resonance image showing vaginal cuff recurrence

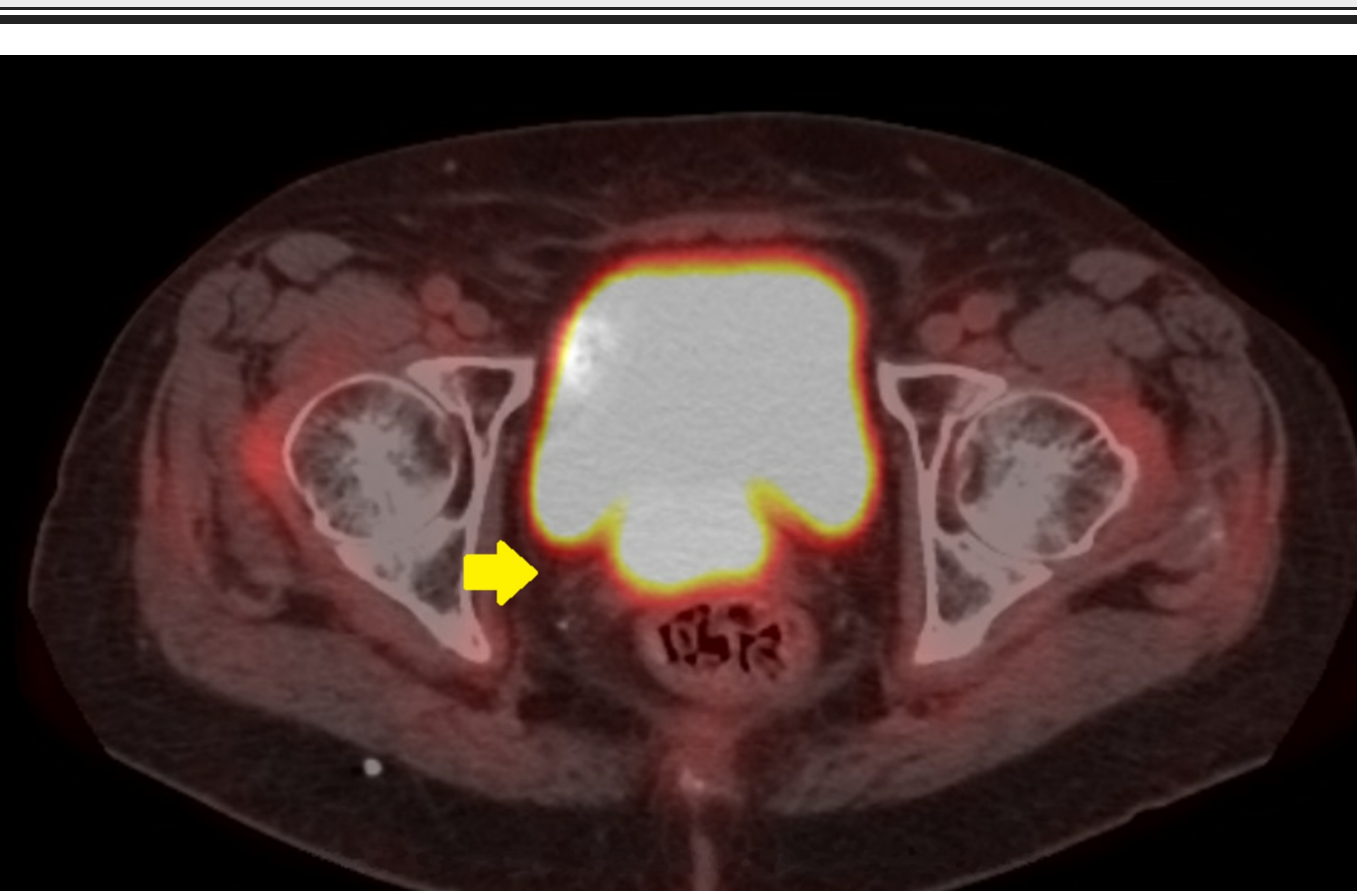

FIGURE 2: Pre-implant axial positron emission tomography image showing vaginal cuff recurrence

After a joint discussion between radiation oncology and gynecologic oncology, the decision was made to place allograft mesh spacer on the superior aspect of the vaginal cuff to protect likely adhered bowel given her surgical history and prior pelvic irradiation. She underwent 


\section{Cureus}

laparoscopy with lysis of adhesions with separation of large bowel from the posterior cul-de-sac and vaginal cuff prior to placement of an dermal matrix spacer (Figure 3). 12 interstitial needles were placed using a Syed-Neblett template intra-operatively during the same case. She underwent CT-based simulation supine with a full bladder. MRI fusion was used to assist in contouring at-risk volumes. The patient completed $30 \mathrm{~Gy}$ in five fractions with twice daily fractionation to the area of recurrent disease. Cumulative dose in $2 \mathrm{~Gy}$ per day equivalent to bladder, bowel, rectum and sigmoid were limited to their respective tolerances (Figure 4, Table 1) [9-11]. The area of recurrence was escalated to $79.9 \mathrm{~Gy}$, with $49.6 \mathrm{~Gy}$ contributed from the Syed implant. The clinical target volume (CTV) was limited by rectal dose, which received a total of 70.8 Gy maximum dose.

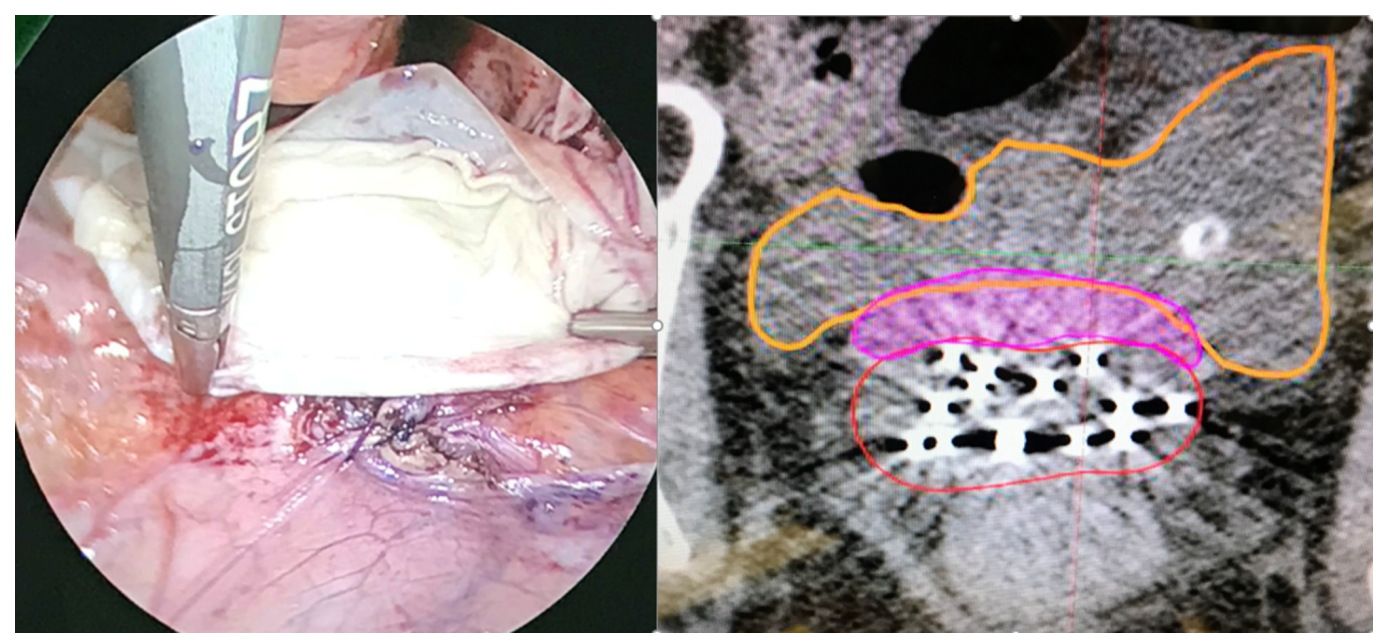

\section{FIGURE 3: Laparoscopic placement of allograft mesh spacer}

Biologic mesh (pink) placed between area of recurrence (red) and bladder (orange). Left: intraoperative photo. Right: simulation computerized tomography.

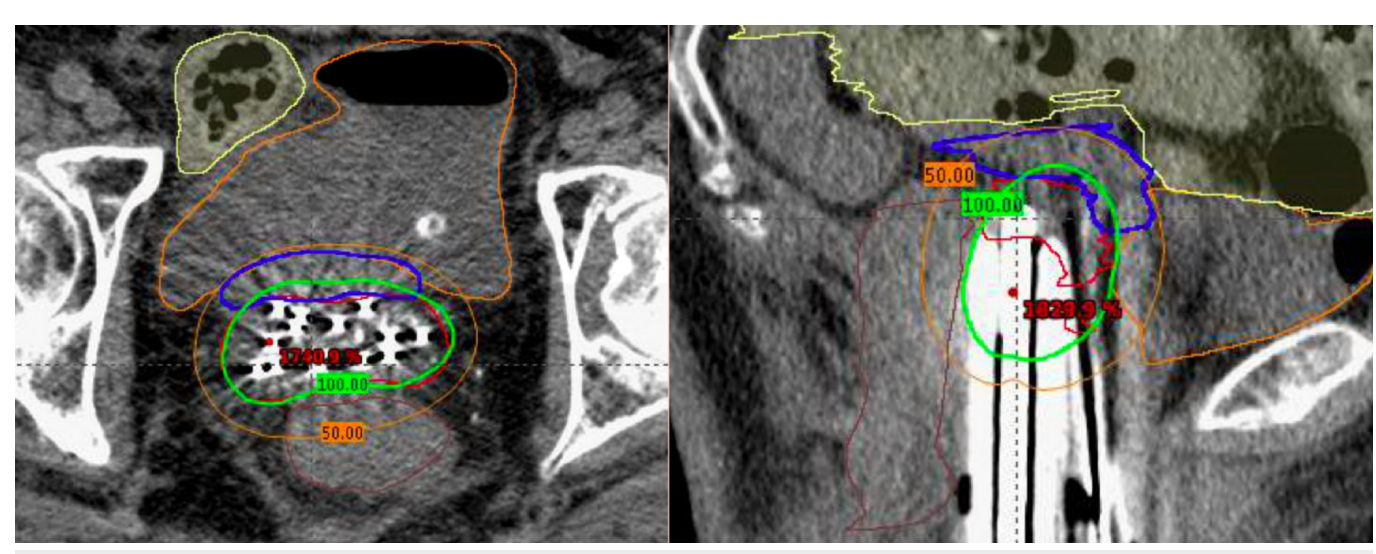

FIGURE 4: Computerized tomography images of final approved dosimetric plan

Left: Axial view. High risk clinical target volume (red) and D100 (green) are separated from the bladder (orange), rectum (brown), and bowel (yellow) by the implanted spacer (blue). Right: Sagittal view. 


\section{Cureus}

\begin{tabular}{|l|lllll|}
\hline & Dose (G) & & & & \\
\hline & Rectum & Bladder & Sigmoid & Bowel & CTV \\
\hline HDR Cylinder & 16.3 & 11.8 & 2.6 & 6.2 & - \\
HDR Syed & 25.2 & 32.3 & 10.1 & 11.3 & 49.6 \\
EBRT & 29.4 & 29.4 & 29.4 & 29.4 & 30.1 \\
Total Equivalent Dose & 70.8 & 73.5 & 42.1 & 46.9 & 79.7 \\
Recc Limit & $<75$ & $<90$ & $<75$ & $<75$ & $>85$ \\
\hline
\end{tabular}

\section{TABLE 1: Cumulative dose calculations in 2 Gy per fraction equivalents}

HDR: High dose rate; EBRT: External beam radiation therapy; CTV: Clinical target volume.

At one-month follow-up, she reported mild but improving dysuria and vaginal soreness with no radiographic evidence of disease (Figures 5, 6). She otherwise denied symptoms of acute toxicity, including diarrhea. Restaging MRI of the pelvis and PET-CT at 12 months demonstrated a complete clinical response.

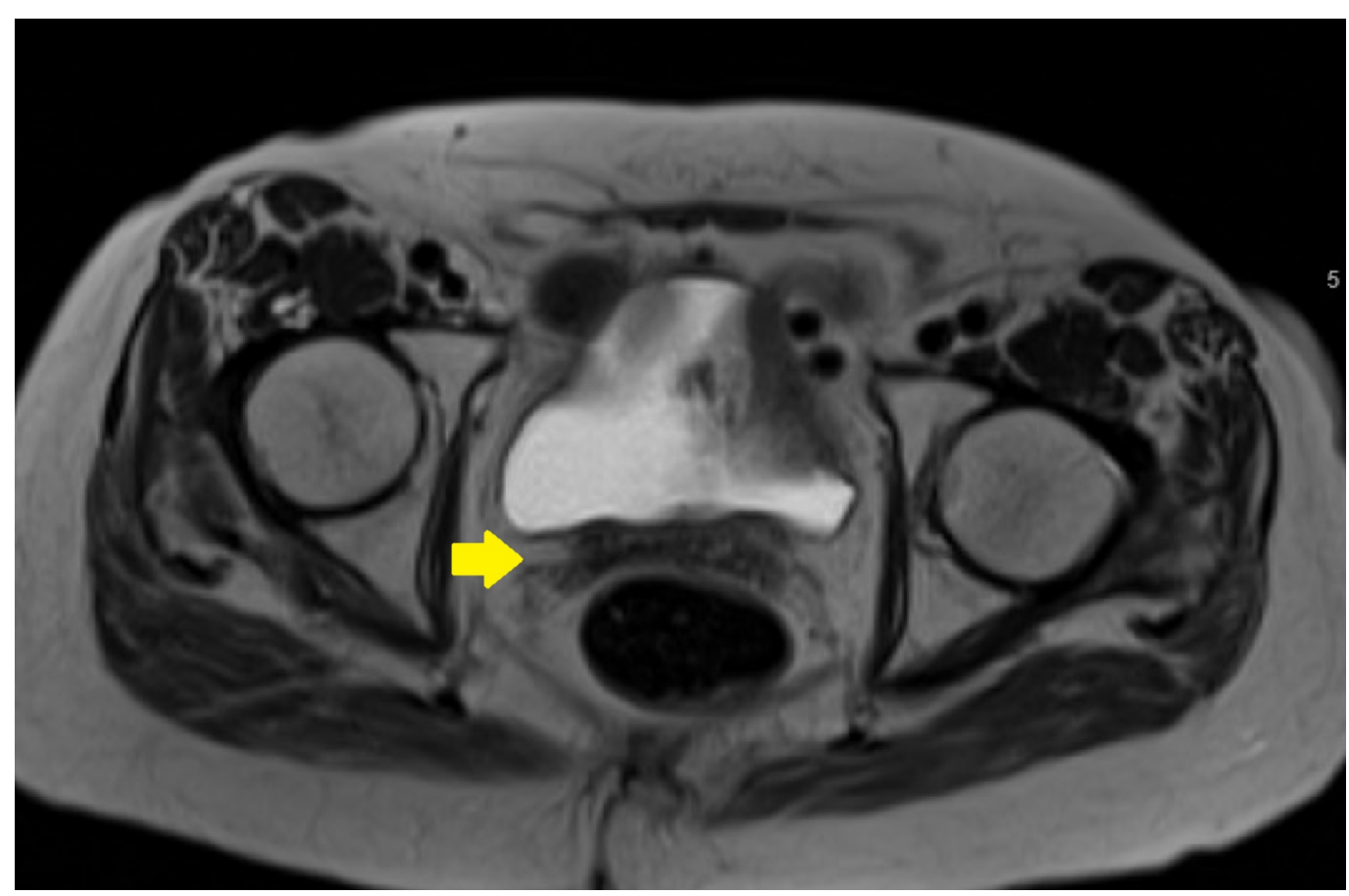

FIGURE 5: One-month post-treatment axial T2 magnetic resonance image 


\section{Cureus}

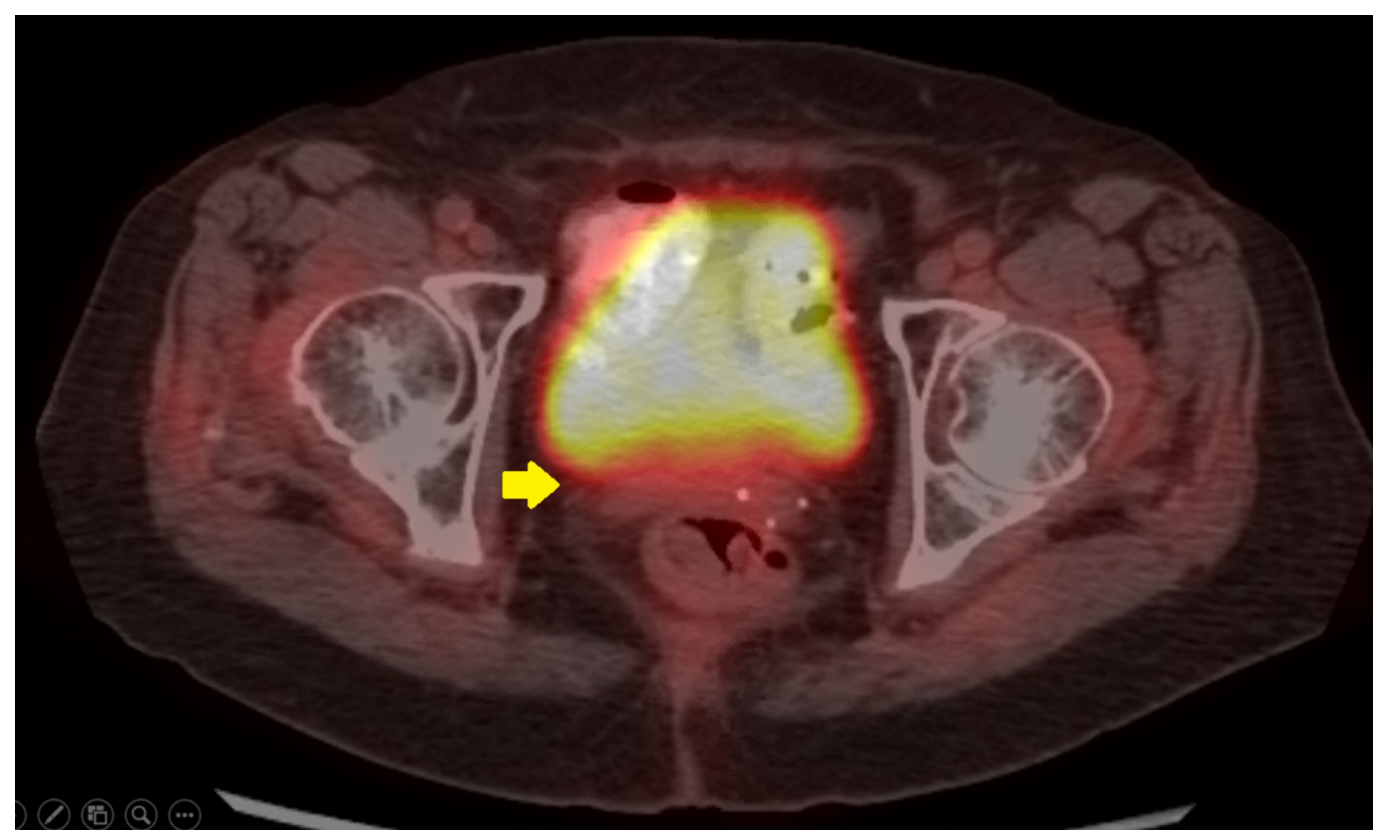

FIGURE 6: One-month post-Syed salvage brachytherapy axial positron emission tomography image

\section{Discussion}

We reported a patient with a solitary vaginal cuff recurrence after previous pelvic radiation successfully treated with re-irradiation using interstitial brachytherapy. Dose optimization was facilitated by an allograft mesh spacer to protect the overlying small bowel and bladder. The need for a spacer in our case was discussed due to the patient's history of previous irradiation and surgery. Allograft mesh is a common material used in general and gynecologic surgery for tissue reinforcement; it was chosen due to its desirable safety profile and relatively low-risk of infection. Laparoscopic technique was utilized given this patient's concurrent need for lysis of adhesions at the time of placement.

Since the first reported radioprotective spacer use in radiation therapy, multiple types have been developed in attempt to minimize high dose to normal tissue, reduce the risk of shortand long-term treatment toxicity, and overall cost of patient care. While no clear standard guidelines or indications exist in the literature for the use of spacers, a multidisciplinary discussion with review of imaging would be helpful in selecting appropriate patients prior to radiation therapy. Patients receiving brachytherapy or stereotactic body radiotherapy with critical structures adjacent to tumor would likely benefit most, especially in the setting of reirradiation.

Spacers can be placed open, laparoscopically, endoscopically, or via injection. Minimally invasive injection can be an option if the anatomic location is amenable. Specialty surgery, interventional radiology, and/or procedural medicine consult may be of assistance as available procedures vary by institution. Approach and need may also be determined by the individual's pre-operative clearance as cancer patients may be too sick to withstand open procedure for a prophylactic benefit to morbidity. Lastly, placement of a surgical implant could be considered at the time of definitive surgery if adjuvant radiation is planned.

Although studies throughout the literature report that spacers are generally safe, patients should be aware of the possible risks associated with the prophylactic placement of a 
radioprotective foreign material [12-20]. Complication risk is related to technique, anatomic site, and the device itself. The implantation of a spacer subjects patients to procedural risks (bleeding, pain, infection, and iatrogenic trauma to nearby structures) in addition to those associated with anesthesia. Local erosion and allergic reaction is possible. Future radiographic interpretation may be confounded by placement of a surgical spacer. Furthermore, tumor dissemination is a theoretical risk associated with manipulation of tissue in proximity with malignancy. However, no deaths have been reported with implanted or injected spacer for radiation therapy. Ultimately, the benefit of achieving ideal dosimetry and decreasing treatment-related morbidity in these patients may outweigh the small risk of an adverse event.

\section{Conclusions}

It can be challenging to achieve optimal dosimetry in the context of re-irradiation in a postoperative patient. In our case, laparoscopically placed allograft mesh spacer was used prior to interstitial brachytherapy to achieve definitive doses to known recurrence while sparing normal tissue.

\section{Additional Information Disclosures}

Human subjects: Consent was obtained by all participants in this study. N/A issued approval N/A. IRB approval not required (exempt). Conflicts of interest: In compliance with the ICMJE uniform disclosure form, all authors declare the following: Payment/services info: All authors have declared that no financial support was received from any organization for the submitted work. Financial relationships: All authors have declared that they have no financial relationships at present or within the previous three years with any organizations that might have an interest in the submitted work. Other relationships: All authors have declared that there are no other relationships or activities that could appear to have influenced the submitted work.

\section{Acknowledgements}

We would like to thank Michelle Quan, PhD; Bin S. Teh, MD, PhD; and Bijal Patel, MS.

\section{References}

1. Dürig M, Steenblock U, Heberer M, Harder F: Prevention of radiation injuries to the small intestine. Surg Gynecol Obstet. 1984, 159:162-163.

2. Niwa K, Morita K, Kanazawa H, Yokoi M: Usefulness of a radiolucent spacer in radiation therapy for cancer of the tongue. (Article in Japanese). Gan No Rinsho. 1984, 30:1861-1865.

3. Tamamoto M, Fujita M, Yamamoto T, Hamada T: Techniques for making spacers in interstitial brachytherapy for tongue cancer. Int J Prosthodont. 1996, 9:95-98.

4. Miura M, Takeda M, Sasaki T, et al.: Factors affecting mandibular complications in low dose rate brachytherapy for oral tongue carcinoma with special reference to spacer. Int J Radiat Oncol Biol Phys. 1998, 41:763-770. 10.1016/S0360-3016(98)00118-7

5. Obinata K, Ohmori K, Tuchiya K, Nishioka T, Shirato H, Nakamura M: Clinical study of a spacer to help prevent osteoradionecrosis resulting from brachytherapy for tongue cancer. Oral Surg Oral Med Oral Pathol Oral Radiol Endod. 2003, 95:246-250. 10.1067/moe.2003.94

6. Murakami S, Verdonschot RG, Kakimoto N, Sumida I, Fujiwara M, Ogawa K, Furukawa S: Preventing complications from high-dose rate brachytherapy when treating mobile tongue cancer via the application of a modular lead-lined spacer. PloS One. 2016, 11:0154226. 10.1371/journal.pone.0154226

7. Damato AL, Kassick M, Viswanathan AN: Rectum and bladder spacing in cervical cancer brachytherapy using a novel injectable hydrogel compound. Brachytherapy. 2017, 16:949-955. 10.1016/j.brachy.2017.04.236 
8. Schutzer ME, Orio PF, Biagioli MC, Asher DA, Lomas H, Moghanaki D: A review of rectal toxicity following permanent low dose-rate prostate brachytherapy and the potential value of biodegradable rectal spacers. Prostate Cancer Prostatic Dis. 2015, 18:96-103. 10.1038/pcan.2015.4

9. Nag S, Gupta N: A simple method of obtaining equivalent doses for use in HDR brachytherapy . Int J Radiat Oncol Biology Physics. 2000, 46:507-513. 10.1016/S0360-3016(99)00330-2

10. ABS consensus statements. (2019). Accessed: July 24, 2019: https://www.americanbrachytherapy.org/consensus-statements/.

11. Pötter R, Tanderup K, Kirisits C, et al.: The EMBRACE II study: the outcome and prospect of two decades of evolution within the GEC-ESTRO GYN working group and the EMBRACE studies. Clin Transl Radiat Oncol. 2018, 9:48-60. 10.1016/j.ctro.2018.01.001

12. Sezeur A, Martella L, Abbou C, et al.: Small intestine protection from radiation by means of a removable adapted prosthesis. Am J Surg. 1999, 178:22-25. 10.1016/S0002-9610(99)00112-9

13. Sezeur A, Abbou C, Chopin D, Rey P, Leandri J: Protection of the small intestine against irradiation by means of a removable prosthesis. ASAIO Trans. 1990, 36:681-683.

14. Sezeur A, Abbou C, Rey P, et al.: New surgical procedure for the protection of the small intestine before postoperative pelvic irradiation. Ann Chir. 1990, 44:352-355.

15. Rai B, Patel FD, Chakraborty S, et al.: Bladder-rectum spacer balloon versus vaginal gauze packing in high dose rate brachytherapy in cervical cancer: a randomised study (part ii). Clin Oncol (R Coll Radiol). 2015, 27:713-719. 10.1016/j.clon.2015.06.014

16. Uhl M, Herfarth K, Eble MJ, et al.: Absorbable hydrogel spacer use in men undergoing prostate cancer radiotherapy: 12 month toxicity and proctoscopy results of a prospective multicenter phase II trial. Radiat Oncol. 2014, 9:96.

17. Uhl M, van Triest B, Eble MJ, Weber DC, Herfarth K, De Weese TL: Low rectal toxicity after dose escalated IMRT treatment of prostate cancer using an absorbable hydrogel for increasing and maintaining space between the rectum and prostate: results of a multi-institutional phase II trial. Radiother Oncol. 2013, 106:215-219. 10.1016/j.radonc.2012.11.009

18. Wilder RB, Barme GA, Gilbert RF, et al.: Cross-linked hyaluronan gel reduces the acute rectal toxicity of radiotherapy for prostate cancer. Int J Radiat Oncol Biol Phys. 2010, 77:824-830.

10.1016/j.ijrobp.2009.05.069

19. Chapet O, Decullier E, Bin S, et al.: Prostate hypofractionated radiation therapy with injection of hyaluronic acid: acute toxicities in a phase 2 study. Int J Radiat Oncol Biol Phys. 2015, 91:730-736. 10.1016/j.ijrobp.2014.11.027

20. Wu SY, Boreta L, Wu A, Cheung JP, Cunha JAM, Shinohara K, Chang AJ: Improved rectal dosimetry with the use of SpaceOAR during high-dose-rate brachytherapy. Brachytherapy. 2018, 17:259-264. 10.1016/j.brachy.2017.10.014 